\title{
Laparoscopic total pancreatectomy with islet autotransplantation for chronic pancreatitis
}

\author{
Alex B. Blair, Richard A. Burkhart, Kenzo Hirose, Martin A. Makary \\ Department of Surgery, Johns Hopkins Hospital, Baltimore, MD, USA \\ Contributions: (I) Conception and design: All authors; (II) Administrative support: MA Makary; (III) Provision of study materials or patients: MA \\ Makary; (IV) Collection and assembly of data: All authors; (V) Data analysis and interpretation: All authors; (VI) Manuscript writing: All authors; (VII) \\ Final approval of manuscript: All authors. \\ Correspondence to: Alex B. Blair, MD. Department of Surgery, Johns Hopkins Hospital, 600 N. Wolfe Street, Baltimore, MD 21287, USA. \\ Email: Ablair9@jhmi.edu.
}

\begin{abstract}
Background: Pain from chronic pancreatitis can be debilitating and have far-reaching personal and societal consequences. These consequences can include patient debilitation, worsening of comorbid conditions, narcotic dependence, and implications for health care policy. A variety of surgical procedures have shown limited efficacy for relieving pain in this cohort of patients, and a highly select subset may benefit from a total pancreatectomy (TP). While a brittle form of diabetes can result from TP alone, when combined with islet cell autotransplantation this procedural complication can be minimized. Further, utilizing a minimally invasive approach may be associated with decreased periprocedural pain and length of hospital stay.

Methods: We describe our experience at a single high-volume center in the United States. We present our preferred preoperative evaluation, our updated operative techniques, and the standard perioperative care required following this complex laparoscopic procedure.

Results: Between 2013 and 2015, there were 20 patients who underwent laparoscopic total pancreatectomy with islet autotransplantation (LTPIAT). Perioperative mortality was $0 \%$.

Conclusions: At a high volume pancreatic center with experienced laparoscopic pancreatic surgeons, LTPIAT is feasible and safe for the management of chronic pancreatitis refractory to prior medical and surgical therapies.
\end{abstract}

Keywords: Laparoscopic pancreatectomy with islet autotransplantation; laparoscopic pancreatectomy; minimally invasive pancreatectomy; chronic pancreatitis; diabetes after pancreatectomy

Received: 29 May 2016; Accepted: 29 June 2016; Published: 22 July 2016.

doi: 10.21037 /jovs.2016.07.05

View this article at: http://dx.doi.org/10.21037/jovs.2016.07.05

\section{Introduction}

Chronic pancreatitis is a debilitating disease characterized by progressive and irreversible destruction of the gland parenchyma. The clinical management of these patients is particularly challenging. The repetitive inflammatory insult to the gland often results in intractable, refractory abdominal pain that produces a poor quality of life despite maximal medical management (1). End-stage pancreatitis is often characterized by heterotopic calcification of the pancreatic ducts that are thought to cause functional obstruction of the exocrine portion of the gland. Procedural approaches to decompress this functional obstruction have been used for decades. Options trialed have included endoscopic decompression, functional operative diversion (i.e., pancreaticojejunostomy), or operative gland extirpation (i.e., pancreatectomy). A recent randomized trial suggests that operative approaches are more effective at relieving pain and more durable without need for repeat intervention than endoscopic approaches (2).

There are multiple surgical approaches to this disease. Functional diversion, often completed through the creation of a longitudinal pancreaticojejunostomy, may alleviate some 
Table 1 Patient demographics and operative outcomes of LTPIAT $(\mathrm{n}=20)$

\begin{tabular}{lc}
\hline Characteristics & Data \\
\hline Patient demographics & 8 \\
Male $(\mathrm{n})$ & $39 \pm 13[21-58]$ \\
Age, mean \pm SD [range] (years) & 17 \\
Prior abdominal operations ( $\mathrm{n})$ & \\
Post-operative outcomes & $430 \pm 98$ \\
Operative time, mean \pm SD (min) & $11 \pm 5[5-27]$ \\
Length of stay, mean \pm SD [range] (days) & 0 \\
Mortality (n) & \\
Insulin requirement (U/day) [\%] & $12[60]$ \\
1-10 & $2[10]$ \\
11-20 & $6[30]$ \\
$>20$ & \\
\hline LTPIAT, laparoscopic total pancreatectomy & with islet \\
autotransplantation.
\end{tabular}

of the exocrine insufficiency seen in chronic pancreatitis. While there are also several endocrine advantages, potential downsides to this approach include leaving much of the native gland in place and failing to adequately address chronic pain issues. This downside also applies to partial pancreatectomy in all of its forms, either with resection of the pancreatic head (with or without duodenal preservation) or resection of the body/tail, risking incomplete pain relief or disease recurrence. Total pancreatectomy (TP) removes the whole gland, eliminating the underlying cause of the pain in the chronic pancreatitis patient. TP was historically avoided as the resulting combination of exocrine dysfunction with brittle endocrine dysfunction was particularly difficult to manage. However with recent improvements in postoperative medical management $\mathrm{TP}$ is a technique used with increasing frequency. Exocrine function is increasingly able to be managed with the assistance of oral enzymatic supplements. In many specialized centers, the resulting endocrinopathy after TP is being mitigated by a technique to preserve beta-cell mass, intraoperative isolation and autotransplation of pancreatic islets. The use of concomitant islet autotransplantation (IAT) has been demonstrated to reduce or eliminate the need for exogenous insulin administration after a TP in many modern series (3).

The first total pancreatectomy with islet autotransplantation (TPIAT) was performed in 1977 at the University of Minnesota (4). Our experience at Johns Hopkins Hospital was first described in 1981 with a series of eight patients with chronic pancreatitis who underwent pancreatectomy and IAT (5). Since that time, advancements in technique have steadily improved the outcomes for this option and our program was re-started in 2010. Laparoscopy, particularly in the setting of benign disease, is being increasingly utilized for pancreatic surgery. Limited pancreatectomy (pancreaticoduodenectomy or distal pancreatectomy), for example, when performed by experienced surgeons has been demonstrated to be safe, produce similar outcomes at equal or lesser cost, and lead to decreased length of hospital stay when compared to open pancreatectomy (6-10). The generalization of these findings to TP appears appropriate (as one of the most technically challenging hurdles in pancreaticoduodenectomy, the pancreaticojejunal anastomosis, is eliminated in TP) and is now being utilized in select centers. Beginning in 2013, we began to offer a laparoscopic approach to all appropriate candidates referred to our institution for TPIAT. We present our preferred preoperative evaluation, our updated operative techniques, and the standard perioperative care required following this complex laparoscopic procedure. Finally we briefly discuss our recent outcomes and areas for future consideration with regards to laparoscopic total pancreatectomy with islet autotransplantation (LTPIAT).

\section{Patient and methods}

\section{Patient selection and workup}

Individuals being considered for LTPIAT at our institution have typically had a long antecedent disease course. All referred candidates were diagnosed with chronic pancreatitis refractory to other therapies with $85 \%$ having undergone prior surgical procedures. Table 1 reviews our population data and postoperative outcomes.

Complete preoperative workup includes clinical history, physical examination, and evaluation of baseline glucose tolerance. We will often receive a plethora of imaging studies to evaluate upon initial presentation, including magnetic resonance imaging (MRI), computed tomography (CT) and endoscopic retrograde cholangiography (ERCP). If the patient has not received a high-quality pancreaticprotocol CT scan we obtain one in the immediate preoperative period. Our exclusion criteria unique to a LTPIAT include patients who are unable to safely undergo laparoscopy or those who already manifest insulin dependent diabetes. 


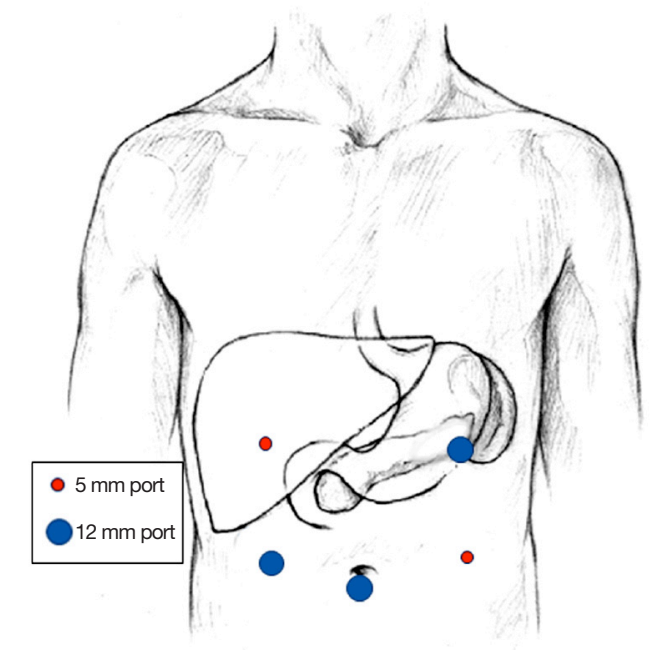

Figure 1 Initial laparoscopic port placement for total pancreatectomy with islet autotransplantation (TPIAT).

\section{Equipment preferences}

Use of a $10-\mathrm{mm}, 45$-degree laparoscope is preferred for optimized visualization. All sutures used during the duration of the procedure are swedged on to the needle to avoid inadvertent loss within the peritoneal cavity. Of note, for all advanced laparoscopic pancreas procedures, a \#10 blade scalpel and Mayo scissors are always available if rapid conversion to open is necessary. Additional device availability is based on surgeon preference including: 30-degree laparoscope, laparoscopic bipolar energy device such as ligaSure, laparoscopic monopolar cautery such as hook dissector, laparoscopic linear stapler, laparoscopic needle driver or endostitch device.

\section{Pre-operative preparation}

Standard perioperative prophylaxis is administered in the preoperative area, including a prophylactic dose of heparin, injected subcutaneously, within 1 hour prior to procedure start and prophylactic antibiotics 30 minutes prior to incision. Preoperative organization and identification of team roles is a key factor in ensuring a smooth and safe procedure. Prior to the day of surgery the availability of the islet cell isolation team is coordinated appropriately. The patient is positioned supine with arms out. Large-bore peripheral intravenous access and a radial arterial line are placed with additional central venous catheter insertion left to the discretion of the operating surgeon and anesthesiology team.
The patient is secured to the table at the thighs and chest, ensuring safety as the operating table may need to be tilted during the procedure to aid in laparoscopic visualization. Preoperative imaging is immediately accessible throughout the operation. Prior to incision, a routine checklist is completed conforming to all institutional and WHO standards.

\section{Role of team members}

The primary surgeon and assistant stand on opposite sides of the table and control the four working instruments, rotating sides based on optimal visualization. A second surgical assistant holds and controls the camera. The laboratory pathology team is stationed in the operating room with a sterile hood for preparation, isolation and purification of islet cells for re-implantation. Endocrinology and pain management consultation teams are used liberally postoperatively to assist with management of blood glucose levels and pain respectively.

\section{Procedure}

Our preferred method for obtaining entry into the abdomen is via a Hassan approach just inferior to the umbilicus. Here a $12 \mathrm{~mm}$ port is placed and pneumoperitoneum is established to a maximal abdominal pressure of $15 \mathrm{~cm}$ of water. The abdomen is then inspected with a laparoscope and adhesions from prior interventions or manifestation of severe pancreatitis are noted. These are taken down sharply and additional laparoscopic ports are placed sequentially as permitted by exposure. In sum, we will typically use five ports. On the right abdomen, one $5 \mathrm{~mm}$ trocar is inserted in the right upper quadrant and one $12 \mathrm{~mm}$ trocar is inserted in the right mid-abdomen (at the approximate intersection between the mid-clavicular line and a line drawn directly laterally from the umbilicus). On the left this pattern is roughly a mirror image of the right, with the larger $12 \mathrm{~mm}$ port high in the left upper quadrant and the $5 \mathrm{~mm}$ port placed lower (Figure 1).

Dissection begins by taking down the falciform ligament to its insertion into the liver with a LigaSure device. An endostitch device is used to tie the base of the falciform and the tail is brought out through a separate $2 \mathrm{~mm}$ stab incision high in the midline to aid in retraction of the liver. The lesser sac is entered through the gastrocolic ligament and the short gastric vessels are divided along the greater curvature of the stomach in their entirety to the 
left crus of the diaphragm. The posterior adhesions of the stomach to the retroperitoneum are mobilized to expose the anterior surface of the pancreas. The stomach or proximal duodenum can be divided depending on planned approach to reconstruction (we will routinely take the pylorus).

The arterial anatomy supplying the liver and head of the pancreas is identified as it runs superior and posterior to the antrum of the stomach. The common hepatic artery, gastroduodenal artery (GDA), and proper hepatic artery are dissected along their course through this region and visualized. The GDA is then skeletonized circumferentially and prepared for transection with clips or stapling device. The common bile duct is dissected circumferentially and the cystic duct is identified to facilitate cholecystectomy.

We then create a tunnel behind the gland and over the portal vein, similar to that done in open surgery. At this point, we will frequently divide the neck or body of the gland to enhance visualization and allow safe dissection of the pancreatic head and uncinate. The remainder of the dissection can be carried out as allowed by patient anatomy and operative positioning. The superior aspect of the pancreas is dissected along the neck and body of the gland to identify the splenic artery takeoff proximal to its course towards the pancreatic tail. The inferior border of the pancreas is then dissected and mobilized out of the retroperitoneum. The splenic vein laterally is identified and dissected free if able at this point. Attention is taken to identify and preserve the inferior mesenteric vein. The superior mesenteric vein's confluence with the splenic vein to form the portal vein is identified during the course of this dissection. The splenic artery and vein are transected between clips or a stapling device and the tail and spleen are dissected free using electrocautery. Finally to mobilize the gland, a laparoscopic Kocher maneuver is completed and a defect in the ligament of Trietz is created in order to deliver the jejunum up through to the superior part of the abdomen.

The head and uncinate is then separated from lateral aspect of the portal vein and the superior mesenteric artery by a combination of blunt dissection, electrocautery, and transection between clips. Approximately $20 \mathrm{~cm}$ of jejunum is delivered through the ligament of Treitz and the jejunum is divided with a laparoscopic stapling device. The small bowel mesentery is divided adjacent to the bowel wall and in the proximal direction toward the uncinate. This plane is carried along towards the superior mesenteric vein and the superior mesenteric artery margin until the specimen is free.

These specimens are extracted in an endocatch bag through an extension of the periumbilical $12 \mathrm{~mm}$ Hassan port's incision. The specimen is then passed off to the intraoperative laboratory team where the GDA and pancreatic duct are cannulated; the gland is distended with collagenase, and subsequently digested. Islet purification is carried out, at our institution, under sterile conditions in the same operating room. A good harvest is critical as transplanted cells cannot divide or replicate. When able, the pancreatic tissue is kept on ice to preserve the function of the remaining islet cells. Minimizing warm ischemia time increases the viability of islets (11), which is positively correlated with insulin independence after TPIAT (12).

While the specimen is being processed the operation continues at the patient bedside. We close the mini-laparotomy in interrupted fashion, leaving two sutures untied to facilitate re-insertion of our $12 \mathrm{~mm}$ port without excessive air leak. Our preferred method of reconstruction has been described in detail previously and includes hepaticojejunostomy with interrupted absorbable suture (13). A stapled side-to-side, antecolic, retrogastric gastrojejunostomy is performed with a common channel created by a single fire of a $60 \mathrm{~mm}$ endoscopic stapling device. The gastrostomy and jejunostomy are then closed with interrupted absorbable suture. In selected patients, a Braun jejunojejunostomy is added in an attempt to mitigate bile acid gastritis.

Once the solution containing pancreatic islet cells is ready for autotransplantation, a hollow-bore 16 gauge needle with intravenous tubing attached is introduced into the abdomen through a $12-\mathrm{mm}$ port site. The needle is placed into the portal vein and the solubilized islets are infused into the liver over a period of 20-45 minutes (Figure 2). Direct pressure applied to the puncture site will often be all that is required to achieve hemostasis. In some cases, a single $5-0$ prolene suture is used to close the venotomy. Peritoneal drains are not required following TP but can be placed through either the left and/or right $5 \mathrm{~mm}$ port site at the discretion of the operating surgeon (Figure 3).

\section{Post-operative management}

Following LTPIAT, most patients are extubated in the operating room after port-site closure. They are admitted to the surgical intensive care unit overnight with careful attention paid to glucose control. An insulin infusion is often used to keep blood glucose values between 100 and $150 \mathrm{mg} / \mathrm{dL}$. A nasogastric tube and arterial pressure line are maintained during the first postoperative night. Typically these patients are stable for transfer to a general surgical 


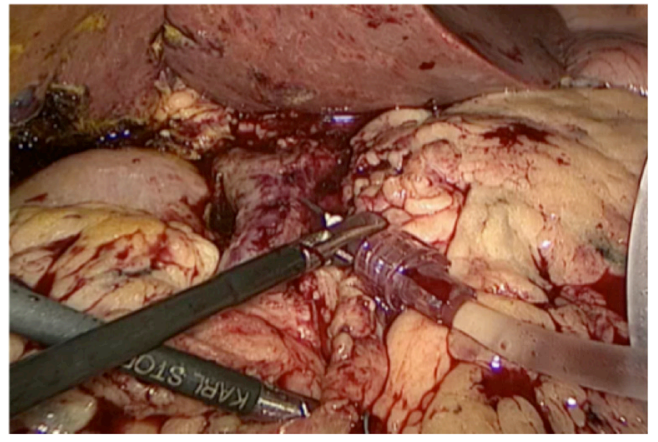

Figure 2 Laparoscopic placement of needle into portal vein for infusion of isolated islet cells.

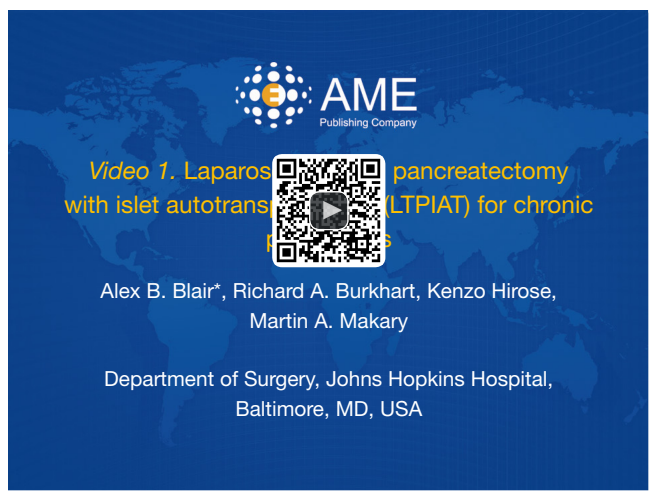

Figure 3 Laparoscopic total pancreatectomy with islet autotransplantation (LTPIAT) for chronic pancreatitis (14). Available online: http://www.asvide.com/articles/1047

floor the following morning. Limited sips and chips diet is often initiated post operatively day 1 with slow advancement towards a goal diet of carbohydrate-limited regular food over the first week. Insulin requirements are aggressively managed over the first few days as the diet is advanced. An extensive education program, started preoperatively, is continued during the patient's hospital stay. Typically, patients require supplemental insulin injections for approximately one-month postoperatively.

\section{Discussion}

Over the last decade, a laparoscopic approach to pancreatectomy has gained popularity with increased adoption in multiple centers across the world. Studies suggest the minimally invasive approach is not only feasible but equivalently efficacious and safe: with similar perioperative morbidity and mortality (6-10,15-19). In particular, the minimally invasive approach to distal pancreatectomy has been widely accepted and clearly demonstrated advantages such as reduced blood loss, reduced overall complication rate, reduced surgical site infection and shorter hospital length of stay (7-10,17). Laparoscopic TP remains a rare procedure, but has shown recent increased use in high volume centers and similarly shown to be safe and feasible $(15,16,18,19)$. A comprehensive review of our initial experience revealed those receiving LTPIAT had significantly shorter hospitalization with median length of stay being 10.5 compared to 14 days for open surgery as well as significant decrease in median postoperative dose of opiate on discharge in the laparoscopic group compared to open (16). With addition of additional patients to this series we have continued to show LTPIAT to be safe and efficacious with a $0 \%$ mortality and evidence of endocrine function preservation with $60 \%$ of patients postoperatively requiring $<10$ units of insulin a day (Table 1 ). Further detailed explanation of our experience is beyond the scope of this technical document and is to be described in a future manuscript. Additional studies are needed to further identify clinically significant outcomes between open and LTPIAT in a larger cohort of patients. Multicenter prospective data would be of benefit due to the limited yearly number of LTPIAT performed.

The learning curve and technical demands of LTPIAT limits accessibility to the hepatobiliary surgeon with advanced laparoscopic skills and isolated to high volume regional centers with the resources to offer multidisciplinary postoperative care and education to these complex patients. These limitations may impede widespread adoption of this technique.

\section{Conclusions}

At a high volume pancreatic center with experienced laparoscopic surgeons LTPIAT is feasible and safe for the management of chronic pancreatitis. As minimally invasive hepatobiliary surgery becomes more widespread, advantages of this technique may be further illuminated.

\section{Acknowledgements}

We extend appreciation to the Division of Gastroenterology and Hepatology, the Division of Endocrinology, Diabetes and Metabolism and the Division of Pathology at Johns Hopkins Hospital for their assistance in multidisciplinary management of the chronic pancreatitis patients undergoing LTPIAT. 


\section{Footnote}

Conflicts of Interest: The authors have no conflicts of interest to declare.

Ethical Statement: The study was approved by Johns Hopkins Hospital institutional review board and informed consent was obtained from the patient for intraoperative filming of surgery. Outcomes discussion is a retrospective review of deidentified information in our institution's secure pancreatic database.

\section{References}

1. Bornman PC, Marks IN, Girdwood AW, et al. Pathogenesis of pain in chronic pancreatitis: ongoing enigma. World J Surg 2003;27:1175-82.

2. Cahen DL, Gouma DJ, Nio Y, et al. Endoscopic versus surgical drainage of the pancreatic duct in chronic pancreatitis. N Engl J Med 2007;356:676-84.

3. Argo JL, Contreras JL, Wesley MM, et al. Pancreatic resection with islet cell autotransplant for the treatment of severe chronic pancreatitis. Am Surg 2008;74:530-6; discussion 536-7.

4. Najarian JS, Sutherland DE, Matas AJ, et al. Human islet autotransplantation following pancreatectomy. Transplant Proc 1979;11:336-40.

5. Cameron JL, Mehigan DG, Broe PJ, et al. Distal pancreatectomy and islet autotransplantation for chronic pancreatitis. Ann Surg 1981;193:312-7.

6. Song KB, Kim SC, Hwang DW, et al. Matched CaseControl Analysis Comparing Laparoscopic and Open Pylorus-preserving Pancreaticoduodenectomy in Patients With Periampullary Tumors. Ann Surg 2015;262:146-55.

7. Fox AM, Pitzul K, Bhojani F, et al. Comparison of outcomes and costs between laparoscopic distal pancreatectomy and open resection at a single center. Surg Endosc 2012;26:1220-30.

8. Ricci C, Casadei R, Taffurelli G, et al. Laparoscopic Distal Pancreatectomy in Benign or Premalignant Pancreatic Lesions: Is It Really More Cost-Effective than Open

doi: 10.21037 /jovs.2016.07.05

Cite this article as: Blair AB, Burkhart RA, Hirose K, Makary MA. Laparoscopic total pancreatectomy with islet autotransplantation for chronic pancreatitis. J Vis Surg 2016;2:121.
Approach? J Gastrointest Surg 2015;19:1415-24.

9. He J, Pawlik TM, Makary MA, et al. Laparoscopic pancreatic surgery. Minerva Chir 2014;69:371-378.

10. Venkat R, Edil BH, Schulick RD, et al. Laparoscopic distal pancreatectomy is associated with significantly less overall morbidity compared to the open technique: a systematic review and meta-analysis. Ann Surg 2012;25 5:1048-59.

11. Corlett MP, Scharp DW. The effect of pancreatic warm ischemia on islet isolation in rats and dogs. J Surg Res 1988;45:531-6.

12. Ahmad SA, Lowy AM, Wray CJ, et al. Factors associated with insulin and narcotic independence after islet autotransplantation in patients with severe chronic pancreatitis. J Am Coll Surg 2005;201:680-7.

13. Edil BH, Cooper MA, Makary MA. Laparoscopic pancreaticojejunostomy using a barbed suture: a novel technique. J Laparoendosc Adv Surg Tech A 2014;24:887-91.

14. Blair AB, Burkhart RA, Hirose K, et al. Laparoscopic total pancreatectomy with islet autotransplantation (LTPIAT) for chronic pancreatitis. Asvide 2016;3:285. Available online: http://www.asvide.com/articles/1047

15. Sa Cunha A, Rault A, Beau C, et al. A single-institution prospective study of laparoscopic pancreatic resection. Arch Surg 2008;143:289-95; discussion 295.

16. John GK, Singh VK, Pasricha PJ, et al. Delayed Gastric Emptying (DGE) Following Total Pancreatectomy with Islet Auto Transplantation in Patients with Chronic Pancreatitis. J Gastrointest Surg 2015;19:1256-61.

17. Kooby DA, Gillespie T, Bentrem D, et al. Left-sided pancreatectomy: a multicenter comparison of laparoscopic and open approaches. Ann Surg 2008;248:438-46.

18. Casadei R, Marchegiani G, Laterza M, et al. Total pancreatectomy: doing it with a mini-invasive approach. JOP 2009;10:328-31.

19. Choi SH, Hwang HK, Kang CM, et al. Pylorus- and spleen-preserving total pancreatoduodenectomy with resection of both whole splenic vessels: feasibility and laparoscopic application to intraductal papillary mucin-producing tumors of the pancreas. Surg Endosc 2012;26:2072-7. 